NOTICE: This is the author's version of a work that was accepted for publication in Hydrometallurgy. Changes resulting from the publishing process, such as peer review, editing, corrections, structural formatting, and other quality control mechanisms may not be reflected in this document. Changes may have been made to this work since it was submitted for publication. A definitive version was subsequently published in Hydrometallurgy, Vol. 147-148. (2014).

http://doi.org/10.1016/i.hydromet.2014.06.002 


\title{
Investigating the impact of anatase on the dissolution of boehmite
}

\author{
M. Ireland ${ }^{\ddagger}$ X. Wang\#, T. Radomirovic ${ }^{\ddagger}$, P. Smith^, F. Jones ${ }^{\ddagger *}$ \\ *Corresponding author; Chemistry department, Curtin University, GPO Box U1987, Perth WA 6845 Australia. Phone: \\ 6189266 7677. Email: F.Jones@ curtin.edu.au \\ \# John de Laeter Centre, Curtin University, GPO Box U1987, Perth WA 6845 Australia \\ \$ Chemistry department, Curtin University, GPO Box U1987, Perth WA 6845 Australia \\ $\wedge$ CSIRO Process Science and Engineering, Waterford
}

\begin{abstract}
The dissolution of boehmite in synthetic Bayer liquors is inhibited in the presence of anatase. It has been suggested both by us and others (eg. Loan et al., 2005), that dissolved titanate adsorbs onto the surface of the boehmite limiting its dissolution. This manuscript reports on digestion, X-ray diffraction (XRD) and transmission electron microscopy (TEM) investigations of boehmite solids partially dissolved in synthetic Bayer liquor in the presence of anatase. Energy filtered transmission electron microscopy (EFTEM) is used to obtain elemental distribution maps, which support the adsorption hypothesis and show enrichment of titanium on the boehmite crystals. Interestingly, the aluminium also becomes associated with some of the Ti-rich particles.
\end{abstract}

Keywords: Bayer process; dissolution; boehmite; anatase; sodium titanate; EFTEM

\subsection{Introduction}

The Bayer process used to extract alumina from bauxite involves leaching the bauxite with sodium hydroxide solutions at elevated temperatures and pressures (Autier-Martin et al., 2001; Pearson, 1955) followed by separating the insoluble solid impurities from the pregnant liquor, re-precipitating pure gibbsite, and removing water by calcination to produce alumina. The alumina bearing minerals most commonly found in bauxite are gibbsite $\left(\mathrm{Al}(\mathrm{OH})_{3}\right.$ also called alumina tri-hydrate) and boehmite $(\gamma \mathrm{AlOOH}$, also called alumina mono-hydrate), while diaspore (an alternative alumina mono-hydrate, $\alpha \mathrm{AlOOH}$ ) is less common (Hudson et al. 2000). The temperature used in the digestion stage of alumina refining varies according to the main aluminium bearing phase; gibbsite can be digested at $140-150^{\circ} \mathrm{C}$, while boehmite requires temperatures above $\sim 220^{\circ} \mathrm{C}$ (typically 
$250^{\circ} \mathrm{C}$ ). Ti-bearing minerals have been found to slow the extraction of boehmite at high temperatures (Authier-Martin et al., 2001); with the phase being important - anatase has a significant impact on dissolution, while rutile showed no effect. It has been suggested that at high temperatures, the titanium reacts with $\mathrm{NaOH}$ to form sodium titanates forming a gelatinous film that coats the boehmite particles and impedes their digestion (Loan et al., 2005, Whittington, 1996). Whittington (Whittington, 1996) found that the effect of sodium titanate on extraction is not seen at low temperature digestions $\left(\sim 180^{\circ} \mathrm{C}\right)$ and stated that anatase does not react in caustic until $\sim 180^{\circ} \mathrm{C}$, thereby suggesting why gibbsite extraction is unaffected. However, as Dudek et al., (2009) found, anatase can have a non-trivial impact on boehmite dissolution even at temperatures as low as $90^{\circ} \mathrm{C}$. Lime $\left(\mathrm{CaO}\right.$ or $\left.\mathrm{Ca}(\mathrm{OH})_{2}\right)$, added to Bayer liquor, is proposed to minimise the formation of sodium titanate by reacting with titanate in solution to form perovskite $\left(\mathrm{CaTiO}_{3}\right)$ which is highly insoluble and is removed with the red mud (Loan et al., 2005; Shultze-Ronhof et al., 1972).

A previous study (Chester et al., 2009) found that the extent of dissolution for anatase and rutile are similar at $90^{\circ} \mathrm{C}$ (after 24 hours) while sodium titanate dissolved least of the three minerals when aluminate ions are present. For all three Ti-bearing solids the amount dissolved is generally related to the free caustic (FC, defined below). Only at higher aluminate concentrations (lower FC values) was anatase dissolution found not to be related to the FC and it was suggested that this was due to the precipitation of an amorphous and impervious layer (possibly of sodium titanate). Another study (Dudek et al., 2009) on the effect of anatase, rutile and sodium titanate on the dissolution of boehmite and gibbsite at $90^{\circ} \mathrm{C}$ found that Ti-bearing minerals had no effect on the digestion of gibbsite at $90^{\circ} \mathrm{C}$. Ti-bearing minerals were found to effect boehmite dissolution even at $90^{\circ} \mathrm{C}$, with anatase reducing extraction significantly, followed by sodium titanate and rutile.

In this work, the solids remaining after the partial dissolution of boehmite in the presence of anatase at $250^{\circ} \mathrm{C}$ are investigated. This temperature is characteristic of boehmitic bauxite digestion and, therefore, the results in this study are relevant to current industrial practice. The aim was to investigate the hypothesis that titanates could adsorb onto the surface of the boehmite and limit extraction. In order to achieve this, solids were 
collected, ultramicrotomed and energy-filtered transmission electron microscopy (EFTEM) was used to image the elemental distributions within and on the surface of particles at sub-micron scale spatial resolution. This technique has been successfully used previously to investigate the nickel distribution in natural goethite crystals (Landers, 2011) and goethite-silica cementation structure in Western Australian nickel laterite ores (Wang, 2014).

\subsection{Terminology}

North American Bayer industry nomenclature is used to describe the various concentrations in solution.

- Alumina (A) is expressed as $\mathrm{g} / \mathrm{L} \mathrm{Al}_{2} \mathrm{O}_{3}$,

- Free Caustic (FC) is defined as $\mathrm{g} / \mathrm{L}$ free sodium hydroxide,

- Caustic (C) is defined as the FC plus sodium aluminate,

- Soda $(\mathrm{S})$ is $\mathrm{C}$ plus sodium carbonate

where $\mathrm{FC}, \mathrm{C}$ and $\mathrm{S}$ are all expressed as equivalent $\mathrm{g} / \mathrm{L} \mathrm{Na}_{2} \mathrm{CO}_{3}$

The A/C ratio is a rough guide to supersaturation - spent refinery liquors have $\mathrm{A} / \mathrm{C} \sim 0.35$ and pregnant (green) liquors have $\mathrm{A} / \mathrm{C} \sim 0.7$. The "causticisation" of the liquor is expressed by the ratio $\mathrm{C} / \mathrm{S}$. When this ratio is equal to 1 the liquor contains pure hydroxide, lower values indicate the presence of carbonate, which reduces the potential of the liquor to dissolve aluminium (oxy)hydroxides present.

\subsection{Materials and Methods}

Boehmite was produced via the hydrothermal dehydration of gibbsite (Panias et al., 2003; Asimidis et al., 2001) and was assessed as a pure phase using laboratory X-ray Diffraction (XRD). The anatase powder from Sigma Aldrich (99 wt.\%) and the sodium titanate solids from STREM Chemicals have been previously characterised, with details given in Chester et al., (2009). 


\subsection{XRF Analysis of Boehmite and Anatase}

The "grab" weight method was used, whereby approximately $0.3 \mathrm{~g}$ of accurately weighed and oven dried samples were placed into $95 \% \mathrm{Pt} / \mathrm{Au}$ crucibles with $0.6 \mathrm{~g}$ of 12:22 lithium metaborate/tetraborate flux. The mixture was fused into a homogeneous glass over an oxy-propane flame at a temperature of approximately $1050^{\circ} \mathrm{C}$ and the molten material was poured into a $32 \mathrm{~mm}$ diameter $95 \% \mathrm{Pt} / \mathrm{Au}$ mould heated to a similar temperature. Air jets then cooled the melt for approximately 300 seconds. Samples were analysed on a PANalytical PW2404 $4 \mathrm{~kW}$ wavelength dispersive XRF system for $\mathrm{SiO}_{2}$, $\mathrm{Al}_{2} \mathrm{O}_{3}$ and $\mathrm{Na}_{2} \mathrm{O}$ and $\mathrm{TiO}_{2}$.

\subsection{Liquor Preparation}

Caustic solutions were prepared by dissolving sodium hydroxide pellets (188.67 g) and sodium carbonate powder $(30 \mathrm{~g})$ with deionised water, and brought to volume in a volumetric flask $(1 \mathrm{~L})$. The resulting nominal caustic concentration $(\mathrm{C})$ and soda $(\mathrm{S})$ were $250 \mathrm{~g} / \mathrm{L}$ and $280 \mathrm{~g} / \mathrm{L} \mathrm{Na} \mathrm{CO}_{3}$ respectively. The caustic liquors were then titrated using the Connop method (Connop, 1996) to accurately determine the caustic (C) and soda (S) concentrations.

\subsection{Caustic Digestion of boehmite and anatase mixture at $250^{\circ} \mathrm{C}$}

A Gas Fired Reactor (GFR), Figure 1, was used in the digestion of boehmite in caustic liquor. The caustic liquor $(\mathrm{C}=250,150 \mathrm{~mL})$, boehmite $(30 \pm 1 \mathrm{~g})$ and titanium mineral $(0.45 \pm 0.01 \mathrm{~g}$ anatase or $0.56 \pm 0.01 \mathrm{~g}$ sodium titanate) were added to a $250 \mathrm{~mL}$ stainless steel bomb (a control without titanium mineral was also run). The mass of boehmite was chosen such that if all the boehmite were to dissolve an $\mathrm{A} / \mathrm{C}$ ratio of $\sim 0.7$ would be achieved. This was related to the volume of liquor that could be safely placed into the bomb that would allow for expansion on heating. Finally, the mass of anatase and rutile was determined by using slightly higher ratios to the initial boehmite solids than used in previous work at $90^{\circ} \mathrm{C}$ (Dudek, 2009) and matching the number of moles Ti present in both anatase and sodium titanate. The bomb was loaded into the GFR and the appropriate time-temperature profile was selected. The GFR heated the bomb to $250^{\circ} \mathrm{C}$ at $35^{\circ} \mathrm{C} / \mathrm{min}$. It was kept at $250^{\circ} \mathrm{C}$ for the allocated time and then cooled down rapidly to $30^{\circ} \mathrm{C}$ by 
immersing in a continuous shower. When the program completed, the bomb was removed from the GFR. The sample was filtered using a 2 L pressure filtration vessel, and then was subsequently washed with three sequential washings of $800 \mathrm{~mL}$ deionised water. The residue was dried in an oven overnight at $100^{\circ} \mathrm{C}$, cooled and weighed. This process was repeated for 2, 5, 10, 30 and 60 minutes.

Figure 1.near here

\subsection{Titration and ICP-OES Analysis of Liquors}

After filtration, the $\mathrm{A}, \mathrm{C}$ and $\mathrm{S}$ of exit liquors were determined by a standard titration method (Connop, 1996). Exit liquors were also diluted 10 times with caustic solution containing mannitol (to stabilise aluminium) and analysed for $\mathrm{Na}, \mathrm{Al}$, and $\mathrm{Ti}$ by Inductively Coupled Plasma (ICP) with relevant standards.

\subsection{XRD Analysis of Solid Samples}

X-ray diffraction patterns were collected for the initial boehmite and residue samples using a PANalytical ${ }^{\circledR}$ X'pert X-ray APD diffractometer with Co $\mathrm{K}_{\alpha}$ radiation. A $1^{\circ}$ divergence slit and $1^{\circ}$ anti-scatter slit was used. No Soller slit was used and the width of the receiving slit was set as $0.45 \mathrm{~mm}$. The pattern was measured from $5^{\circ}$ to $90^{\circ} 2 \theta$ at a step size of $0.02^{\circ} 2 \theta$ and a step time of 1 second.

\subsection{Transmission Electron Microscopy (TEM), Energy Dispersive Spectroscopy} (EDS), Selective Area Electron Diffraction (SAED), Energy-filtered Transmission

\section{Electron Microscopy (EFTEM)}

The initial samples (boehmite or anatase) were dispersed in deionised water and agitated in an ultrasonic bath for $2 \mathrm{~min}$. A drop of the suspension for each sample was air dried on carbon film supported by a $3 \mathrm{~mm}$ diameter copper grid for TEM investigation. The sample after 60 min caustic digestion was suspended in Spurrs resin (a common fixative for microscopy applications), the composition of which was adjusted to match the Mohs hardness of boehmite ( 4) and cured for over 48 hours. A thin $(<1 \mu \mathrm{m})$ section 
of the resin was prepared by ultramicrotome and transferred onto a $3 \mathrm{~mm}$ diameter copper grid for TEM investigation.

A JEOL ${ }^{\circledR}$ 3000F transmission electron microscope (TEM) operated at $300 \mathrm{kV}$ and equipped with a Gatan ${ }^{\circledR}$ Orius SC1000 CCD camera was used to image particle morphologies and selected area electron diffraction (SAED) patterns. An Oxford Instruments INCA ${ }^{\circledR} 200$ EDS was used to collect Energy Dispersive X-ray Spectra from sample particles. A post-column Gatan ${ }^{\circledR}$ image filter was used to collect Energy-filtered Transmission Electron Microscopy (EFTEM) images. The EFTEM image was generated by choosing only electrons with an energy loss at the core loss edge of a specific element after a proper background subtraction (Mailis et al., 1988).

\subsection{Results and Discussion}

\subsection{Digestion results}

The dissolution of boehmite in the absence of the anatase in pure caustic was determined by both ICP and titration methods. Figure 2 shows the ICP results agreed well with the titration results, though titration did appear to give slightly higher values. However, this effect appears to be systematic. Due to the consistency of the titration and ICP results the subsequent digestion graphs show the average of the values obtained from the two techniques and the difference between the two measurements (ICP versus titration) shown as the variation.

The boehmite dissolves quickly (within 5 minutes) at this loading and temperature. The digestion of boehmite under refinery conditions is much slower, largely because of the difficulty of extracting it from the other insoluble components of the bauxite matrix.

Figure 2. near here

In the presence of anatase (Figure 3), boehmite dissolution is inhibited at short times. At longer times, the $\mathrm{A} / \mathrm{C}$ values are lower at $60 \mathrm{~min}$ in the presence of anatase compared to the pure boehmite system and this would suggest a possible impact on solubility of 
boehmite. However, these values are within the variability of the two measuring techniques and are therefore inconclusive. Thus, the rate at which boehmite dissolves rather than the total amount of boehmite appears to be affected by the presence of anatase at $250^{\circ} \mathrm{C}$. Anatase reducing the rate of boehmite extraction was also observed at $90^{\circ} \mathrm{C}$ (Dudek et al., 2009). The hypothesis for the inhibition of boehmite dissolution in the presence of anatase proposed is that, as anatase dissolves in solution (as titanate), it subsequently re-precipitates onto the boehmite surface as sodium titanate, inhibiting further dissolution. This occurs because the equilibrium solubility of anatase is higher than that of sodium titanate (Chester et al., 2009). Inhibition does not happen when sodium titanate is the source of the titanium in solution. Figure 3 shows that, in this case, the sodium titanate will dissolve only to its equilibrium solubility and will not cause any titanate to re-precipitate on the boehmite particles.

Figure 3. near here

The starting materials and the residues from the digestion experiments for boehmite + anatase were washed, dried and subsequently assessed by powder XRD. Their XRD patterns are shown in Figure 4. Boehmite (PDF\# 04-013-2972) and anatase (PDF\# 00021-1272) peaks were the only phases identified. Interestingly, even at 60 minutes when all of the boehmite should be dissolved according to the digestion results, there is still boehmite left according to the XRD data. Due to the low solubility of anatase, little of these solids will have dissolved by 60 minutes. Thus, the anatase 101 peak can be used to normalise the XRD patterns. When the 1 minute and 60 minute patterns are compared (after normalization) Figure 4(b) is obtained and shows that the intensities of the boehmite peaks reduce as would be expected from its dissolution but are still significant.

However, there is also a small, new peak at $\sim 12^{\circ} 2 \theta$ that is absent from both the pure boehmite pattern and the pure anatase pattern and can be attributed to sodium titanates (PDF\# 00-031-1329). This is shown in more detail in Figure 4(c) where the sodium titanates' 001 reflection is seen to increase with digestion time. 
Figure 4. near here

$\mathrm{Ti}$ in solution was measured during digestion using ICP. In this way, it is possible to compare the dissolution behaviour of anatase and sodium titanate when digested in the presence of boehmite at $250^{\circ} \mathrm{C}$. These results can be seen in Figure 5 .

Figure 5. near here

Interestingly, the level of anatase and sodium titanate dissolved in terms of [Ti] at 60 minutes is very similar to that measured at $90^{\circ} \mathrm{C}$ for this free caustic level (Chester et al., 2008). These values appear to be at equilibrium as they are no longer changing significantly. While some re-precipitation on cooling is expected to occur the significant difference in the measured [Ti] for anatase versus sodium titanate shows this is by a systematic amount and cannot account for the similarity in [Ti] to that found at $90^{\circ} \mathrm{C}$. It is possible the solubility is not altering significantly from 90 to $250{ }^{\circ} \mathrm{C}$. The studies finding that Ti-bearing minerals only become an issue at temperatures $\geq 180^{\circ} \mathrm{C}$ (Whittington, 1996) was assumed to be due to Ti-bearing minerals only dissolving to a significant extent at temperatures $\geq 180^{\circ} \mathrm{C}$ and so these results would conflict with that notion. However, it is possible that these results are not in conflict with each other but that the [Ti] in solution is not a measure of the anatase solubility but is actually a steady state [Ti] level between the real anatase solubility and the rate of the re-precipitation reaction of sodium titanate much like kaolin dissolution to form desilication products.

Finally, investigation as to where the titanate phase is formed was undertaken. The sample at 60 minutes was chosen since this should contain the greatest amount of titanates.

\subsection{TEM characterisation of source materials}

The TEM images of the starting materials anatase and boehmite and the EDS are shown in Figure 6. The size of the anatase crystals is roughly $150 \mathrm{~nm}$. We did note however, some $\mathrm{Si}$ in the anatase from EDS, which we suspect is the surface layer visible 
in the TEM (silicate is often used to stabilise nanoparticles particularly for pigments (World Health Organisation, 2013)). This layer is about $6.5 \mathrm{~nm}$ thick (Figure 6(a)) and the weight percent of silica surface layer was determined to be $\sim 16$ wt.\% of the total particle according to quantitative EDS analysis in Figure 6(e). No aluminium was detectable in the starting anatase material. The direct measurement of the lattice spacing of the anatase crystal, shown in Figure 6(c), matches the d-spacing of the anatase 101 plane.

The boehmite crystals have a more angular shape and are much larger $(1 \sim 3 \mu \mathrm{m})$ than the anatase crystals. The SAED pattern of the edge of the crystal was recorded and matches with the boehmite diffraction pattern. The EDS of the starting material of boehmite does not contain any detectable amount of titanium. The sodium chloride and calcium chloride content are assumed to come from the original gibbsite precursor or the preparation method.

Figure 6. near here

\subsection{EFTEM characterisation of digested mixture}

Figure 7 presents the distribution of aluminium and titanium in the particles of digested boehmite solids in the presence of anatase after $60 \mathrm{~min}$. Figure 7(a) and Figure 7(b) suggests that titanium tends to enrich on the edges of boehmite particles, while Figure 7(c) and Figure 7(d) suggests that aluminium tends to be distributed through the whole of the anatase particle. Additionally, we find that the Ti imaged at the surface of the boehmite particle is not a homogeneous coating, nor is it found on all particles. This difference in behavior may simply be a concentration effect (limited titanate in solution to form a homogeneous sodium titanate layer). Due to the fact that the particles were dried overnight at $100^{\circ} \mathrm{C}$ we make no comment as to the crystallinity of the adsorbed species as this could have altered on drying.

The EFTEM findings are consistent with the XRD phase identification in that sodium titanate appears to form as digestion proceeds. It is suspected that the titanium rich edges 
of the boehmite particles in Figure 7(a,b) are crystallised sodium titanate. Unfortunately, mapping of the sodium distribution was not possible using EFTEM as the core loss edge of sodium is at a high energy loss $(\sim 1072 \mathrm{eV})$ and signal to noise was an issue. An additional EDS spectrum was also collected from the site of Figure 7(a) and is shown in Figure 8. The $\mathrm{Na} \mathrm{K}_{\alpha}, \mathrm{Ti} \mathrm{K}_{\alpha}$ peaks are visible. The quantitative results of $\mathrm{Na}$ and $\mathrm{Ti}$ concentrations shown in Table 1 are consistent with some form of sodium titanate forming. Therefore, the hypothesis that dissolved titanate is forming sodium titanate on the boehmite surface and inhibits dissolution is supported by these findings.

Interestingly, after 60 min digestion, despite most of the aluminium being dissolved into the leachate, it appears that some of this aluminium finds its way to the anatase or Tirich particles. As previously stated, it appears a homogeneous distribution of aluminium is associated with these particles. Other than boehmite, XRD phase identification failed to detect any new aluminium containing phases.

Figure 7. near here

Figure 8. near here

\section{Table 1 near here}

However in Figure 7(c) and (d) there are also Ti rich particles that are associated with Al. Figure 9 and Table 2 shows the EDS results and quantitative analysis of the EDS information from Figure 7c. Perhaps there is a third trace phase being formed for those particles where we see $\mathrm{Ti}$ and $\mathrm{Al}$ associated together, $\mathrm{Al}_{2} \mathrm{TiO}_{5}$, i.e. aluminium titanate, although this is speculative at this stage (this is because, being an ultramicrotomed sample, adsorption should have resulted in particles with $\mathrm{Al}$ enrichment on the rim of the particle not throughout the visible particle). Hence, another possible mechanism for the lower boehmite extraction in the presence of anatase is that aluminium is lost by adsorption/incorporation onto or into Ti-rich particles. 
Figure 9. near here

Table 2. near here

\subsection{Conclusions}

Anatase is seen to inhibit the rate at which boehmite dissolves at $250^{\circ} \mathrm{C}$ confirming that the presence of anatase can limit the extraction of aluminium from boehmite containing bauxites. This is not observed when sodium titanate is present. As previously found at $90^{\circ} \mathrm{C}$, the formed product of anatase dissolution is sodium titanate, confirmed from XRD.

Unexpectedly, the solubility of anatase and sodium titanate at $250^{\circ} \mathrm{C}$ is similar to that at $90^{\circ} \mathrm{C}$ suggesting the possibility that solubility of these titanium compounds is somewhat insensitive to temperature. However, this is not supported by experience where $180{ }^{\circ} \mathrm{C}$ was required before issues with anatase are encountered in real refinery situations. As found at $90^{\circ} \mathrm{C}$, sodium titanate is still the thermodynamic product and any titanate in solution above its equilibrium solubility will re-precipitate.

Finally, EFTEM has shown that the titanate forms an inhomogeneous layer on the boehmite surface. Perhaps more interestingly, the aluminium can also associate with titanium and perhaps be another mechanism by which boehmite extraction is inhibited.

\subsection{Acknowledgements}

The authors acknowledge the facilities, and the scientific and technical assistance of the Australian Microscopy \& Microanalysis Research Facility at the Centre for Microscopy, Characterisation \& Analysis, The University of Western Australia, a facility funded by the University, State and Commonwealth Governments.

\subsection{References}

Asimidis, P., Panias, D., Paspaliaris, I, (2001) "Solubility of boehmite in concentrated sodium hydroxide solutions: model development and assessment" Hydrometallurgy, 59, 15-29. 
Authier-Martin, M., Forte, G., Ostap, S., See, J., (2001) “The mineralogy of bauxite for producing smelter-grade alumina" Journal of Minerals Metals and Materials Society, 53(12) 36-40.

Chester, R., Jones, F., Loan, M., Oliveira, A., Richmond, W. R., (2009) “The dissolution behaviour of titanium oxide phases in synthetic Bayer liquors at $90^{\circ} \mathrm{C} "$ Hydrometallurgy, 96 (3), 215-222

Connop, W.L., (1996) "A New Procedure for the Determination of Alumina, Caustic and Carbonate in Bayer Liquors" Fourth International Alumina Quality Workshop, p. 321-330.

Dudek, K., Jones, F., Radomirovic, T., Smith, P., (2009) "The effect of anatase, rutile and sodium titanate on the dissolution of boehmite and gibbsite at $90^{\circ} \mathrm{C}$ " International Journal of Mineral Processing, 43, (2).

Gow, N. N., Lozej, G. P., (1993) “Bauxite” Geoscience Canada, 20(1), 9-16.

Hudson, L. K., Misra, C., Perrotta, A. J., Wefers, K., \& Williams, C. (2000). Aluminium Oxide, Ullman's Encyclopaedia of Industrial Chemistry (pp. 607-645): Wiley - VCH.

Landers, M., Gräfe, M., Gilkes, R.J., Saunders, M. and Wells, M.A., (2011) "Nickel distribution and speciation in rapidly dehydroxylated goethite in oxide-type lateritic nickel ores: XAS and TEM spectroscopic (EELS and EFTEM) investigation" Aust. J. Earth. Sci., 58(7), 745-765.

Loan, M., Loughlin, B., Haines, J., Croker, D., Fennell, M., Hodnett, B. K., (2005) "In situ time-resolved synchrotron diffraction studies of high temperature Bayer digestions" Alumina Quality Workshop, Perth Australia, 117-122.

Malts, N. S., Poddymov, V. P., Rudashveskii, L. S., Kiselev, V. E., (1985), "The intensifying action of lime upon the kinetics of bauxite leaching" Tsvet. Metal. (English edition), 5, 38-40.

Malis, T., Cheng, S.C. and Egerton, R.F., (1988) "EELS log-ratio technique for specimen-thickness measurement in the TEM" J. Electron Micr. Tech., 8(2), 193200.

Panias, D., Paspaliaris, I., Skoufadis, C, (2003) "Kinetics of boehmite precipitation from supersaturated sodium aluminate solutions" Hydrometallurgy, 68, 57-68. 
Pearson, T. G. In The Chemical Background on the Aluminium Industry, Royal Institute of Chemistry; London, 1955, Monograph no. 3.

Prakash, S., Horvath, Z., (1979), "Behaviour of titanium minerals in the titanium rich Indian bauxite during its digestion by Bayer process" Publ. Techn. Univ. Heavy Industry, Series B., Metallurgy, 34, 43-63.

Shultze-Rhonhof, E., Winkhaus, G., (1972), "Communication on the chemistry of bauxite digestion: 1. Investigations in the system $\mathrm{Na}_{2} \mathrm{O}-\mathrm{CaO}-\mathrm{Al}_{2} \mathrm{O}_{3}-\mathrm{TiO}_{2}-\mathrm{H}_{2} \mathrm{O}$ at $100{ }^{\circ} \mathrm{C}$ and normal pressure" Zeitschrift für anorganische und allgemeine Chemie, 390(2), 97-103.

Wang, X., McDonald, R.G., Hart, R.D., Li, J. and van Riessen, A., (2014) "Acid resistance of goethite in nickel laterite ore from Western Australia. Part II. Effect of liberating cementations on acid leaching performance" Hydrometallurgy, 141, 4958.

Whittington, B.I. 1996. "The chemistry of $\mathrm{CaO}$ and $\mathrm{Ca}(\mathrm{OH})_{2}$ relating to the Bayer process" Hydrometallurgy 43, 13-35

World Health Organisation (WHO), International Agency for Research on Cancer Mongraphs on the Evaluation of Carcinogenic Risks to Humans, vol 93-7, accessed $19^{\text {th }}$ November 2013 (http://monographs.iarc.fr/ENG/Monographs/vo193/mono937.pdf). 
Figure captions

Figure 1. Gas Fired Reactor (LHS, stainless steel bomb mounted horizontally) and an example of a time - temperature profile (not indicative of actual profiles used in the present work - shows heat-up and cool down rates and temperature stability)

Figure 2. Calculated $\mathrm{A} / \mathrm{C}$ from ICP and titration data for boehmite digested in pure caustic (Line drawn to aid reader only). Dissolution of boehmite is complete within 10 minutes and both techniques show similar trends.

Figure 3. Calculated $\mathrm{A} / \mathrm{C}$ from liquor obtained after digestion of boehmite in caustic for pure boehmite, boehmite +anatase and boehmite + sodium titanate. The rate of boehmite dissolution is inhibited when anatase is present but not when sodium titanate is present.

Figure 4. (a) XRD pattern of starting solids before digestion; (b) XRD pattern of 'residues' - solids remaining after boehmite digestion in the presence of anatase at 1 and 60 minutes, the XRD patterns have been normalized to give the same anatase peak heights. Boehmite dissolves with time but does not completely disappear; (c) sodium titanate 001 reflection versus time for solids remaining after boehmite digestion. The sodium titanate peak is increasing with time.

Figure 5. Concentration of $\mathrm{Ti}(\mathrm{mg} / \mathrm{L})$ in solution as measured by ICP for boehmite digestion in the presence of anatase or sodium titanate. Solubility of sodium titanate is lower than that of anatase.

Figure 6. TEM characterisation of the staring materials. (a) TEM image of the anatase crystals; (b) TEM image of some typical boehmite crystals; (c) High resolution TEM images of anatase, 30 planes measured $104.49 \AA$ in the inset, matching with the d-spacing (3.50 ̊) of the anatase 101 plane; (d) SAED from the edge of the boehmite crystals; (e) EDS of the anatase crystals (no aluminium impurity); (f) EDS of the with boehmite crystals (no titanium impurity). Copper signal comes from TEM copper grid.

Figure 7. EFTEM images showing the aluminium and titanium distributions of the residual boehmite particles (a, b) and the residual anatase particles (c, d) after digestion in caustic for 60 minutes. Ti can be found on the surface of boehmite particles while $\mathrm{Al}$ can be found associated with some anatase particles.

Figure 8. $\quad$ EDS of the boehmite particle shown in Figure 7(a).

Figure 9. $\quad$ EDS of the anatase particle shown in Figure 7(c). 

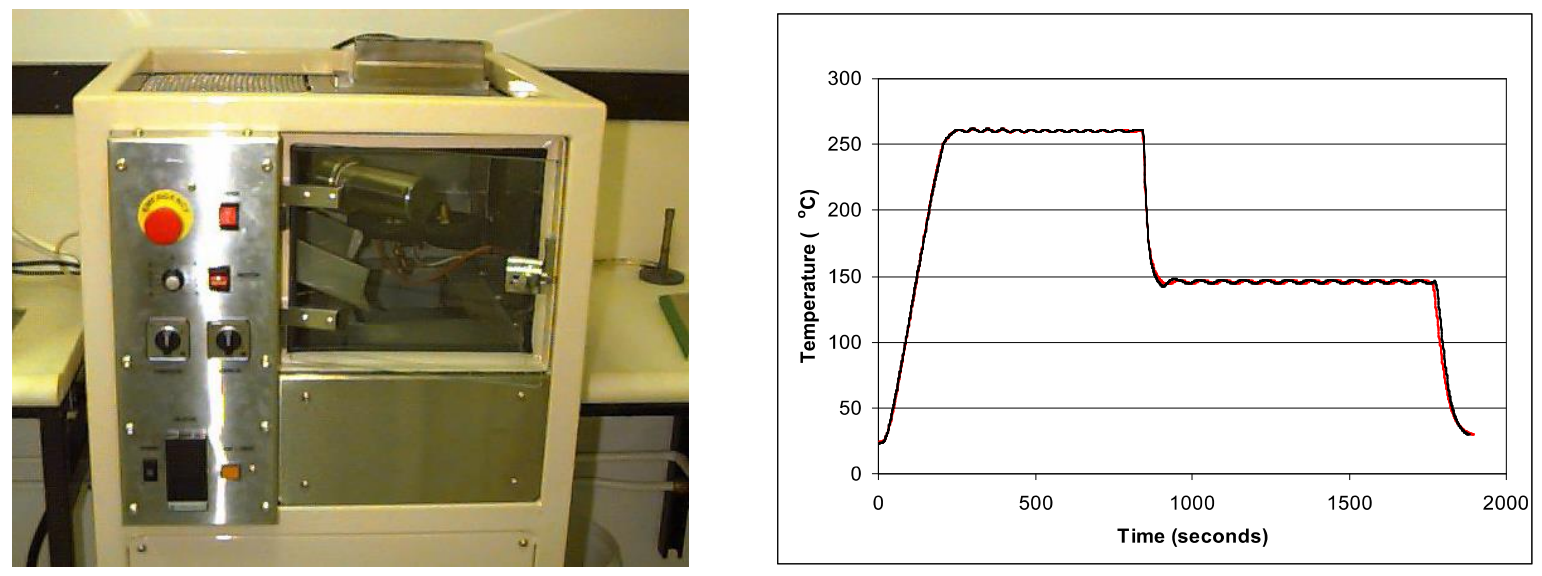

Figure 1. Gas Fired Reactor (LHS, stainless steel bomb mounted horizontally) and an example of a time - temperature profile (not indicative of actual profiles used in the present work - shows heat-up and cool down rates and temperature stability)

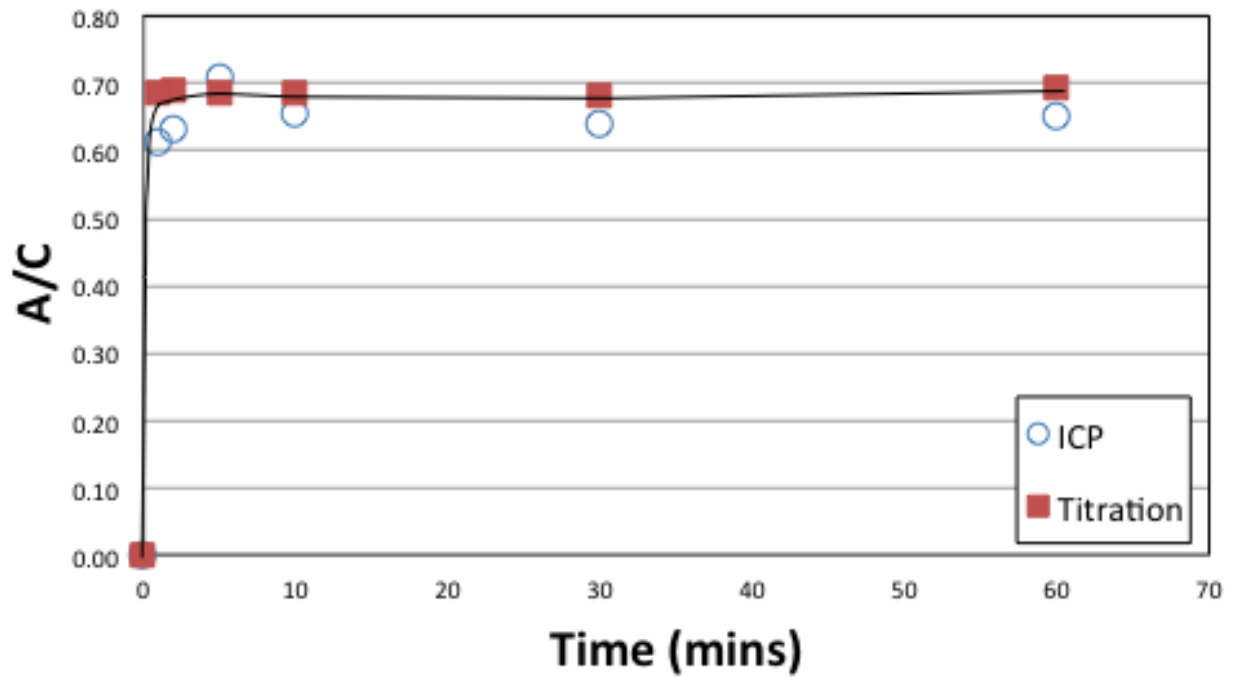

Figure 2. Calculated A/C from ICP and titration data for boehmite digested in pure caustic (Line drawn to aid reader only). Dissolution of boehmite is complete within 10 minutes and both techniques show similar trends. 


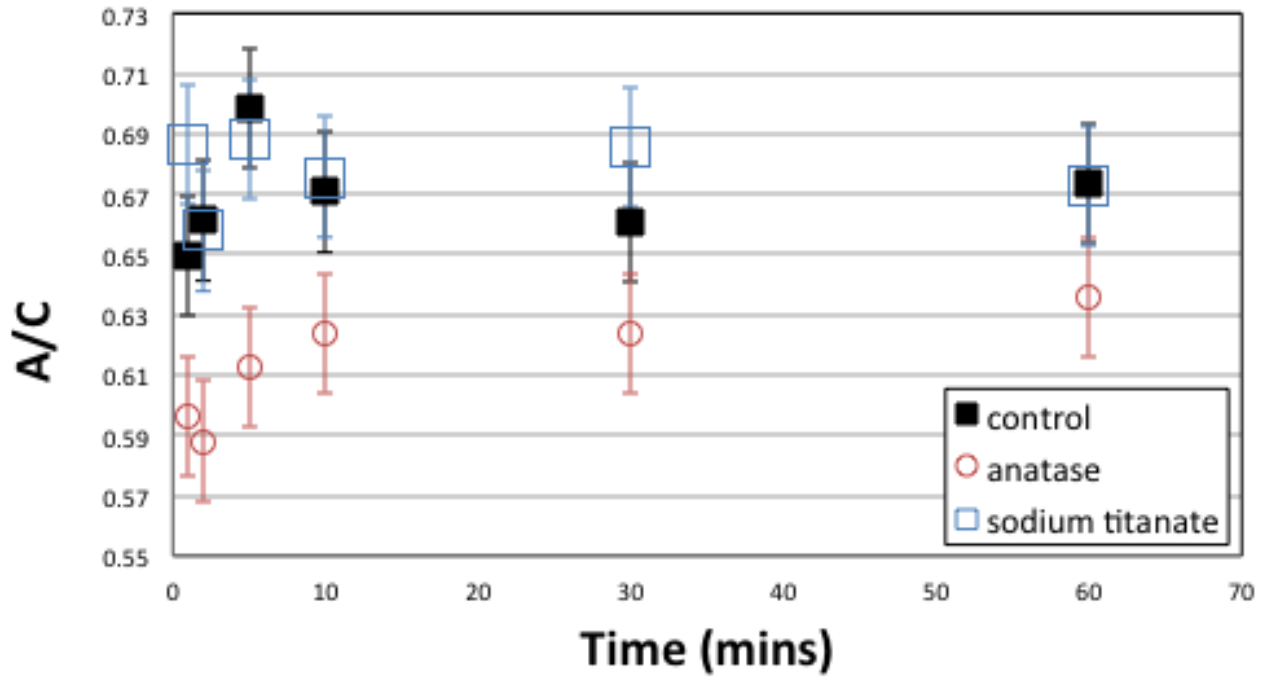

Figure 3. Calculated $\mathrm{A} / \mathrm{C}$ from liquor obtained after digestion of boehmite in caustic for pure boehmite, boehmite + anatase and boehmite + sodium titanate. The rate of boehmite dissolution is inhibited when anatase is present but not when sodium titanate is present.

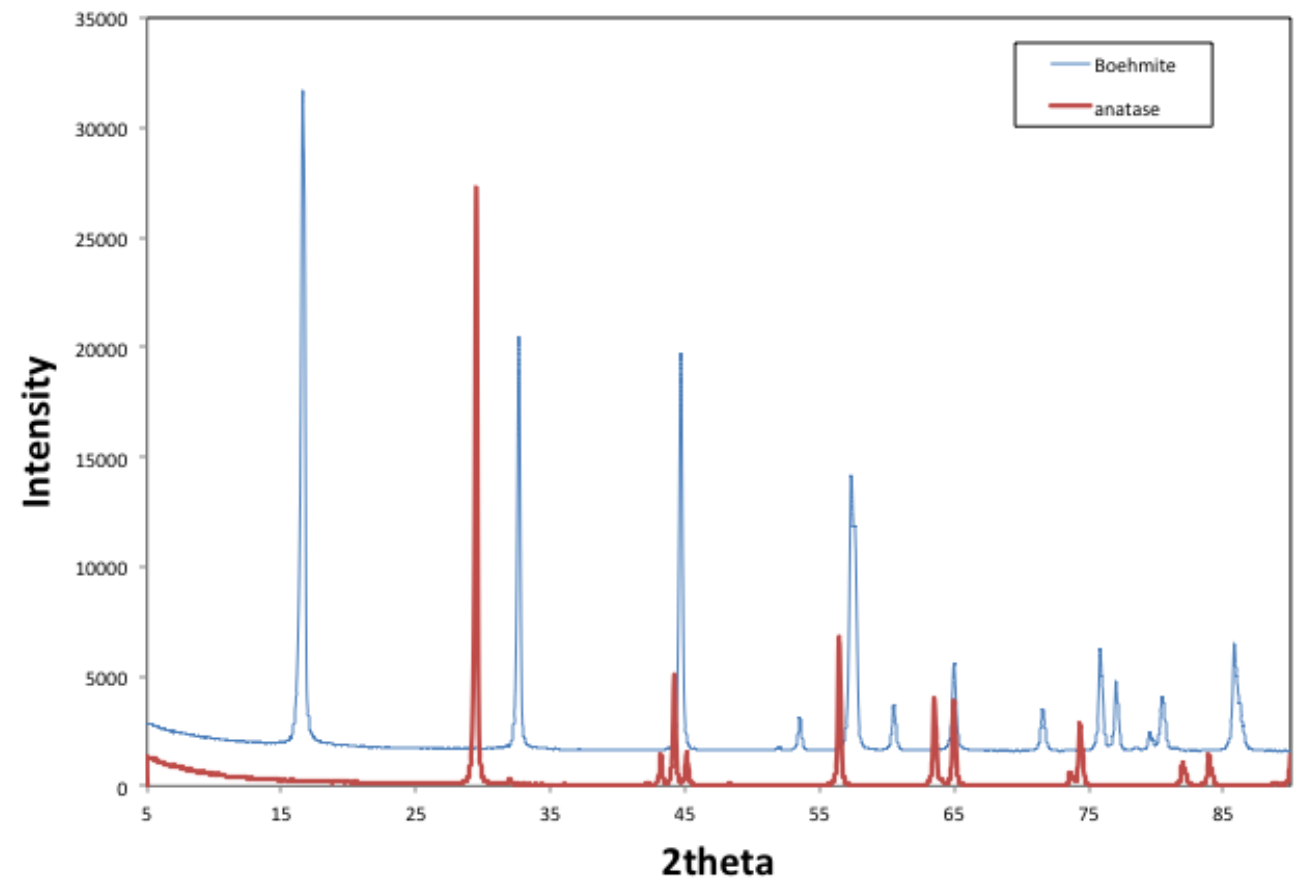

(a) 


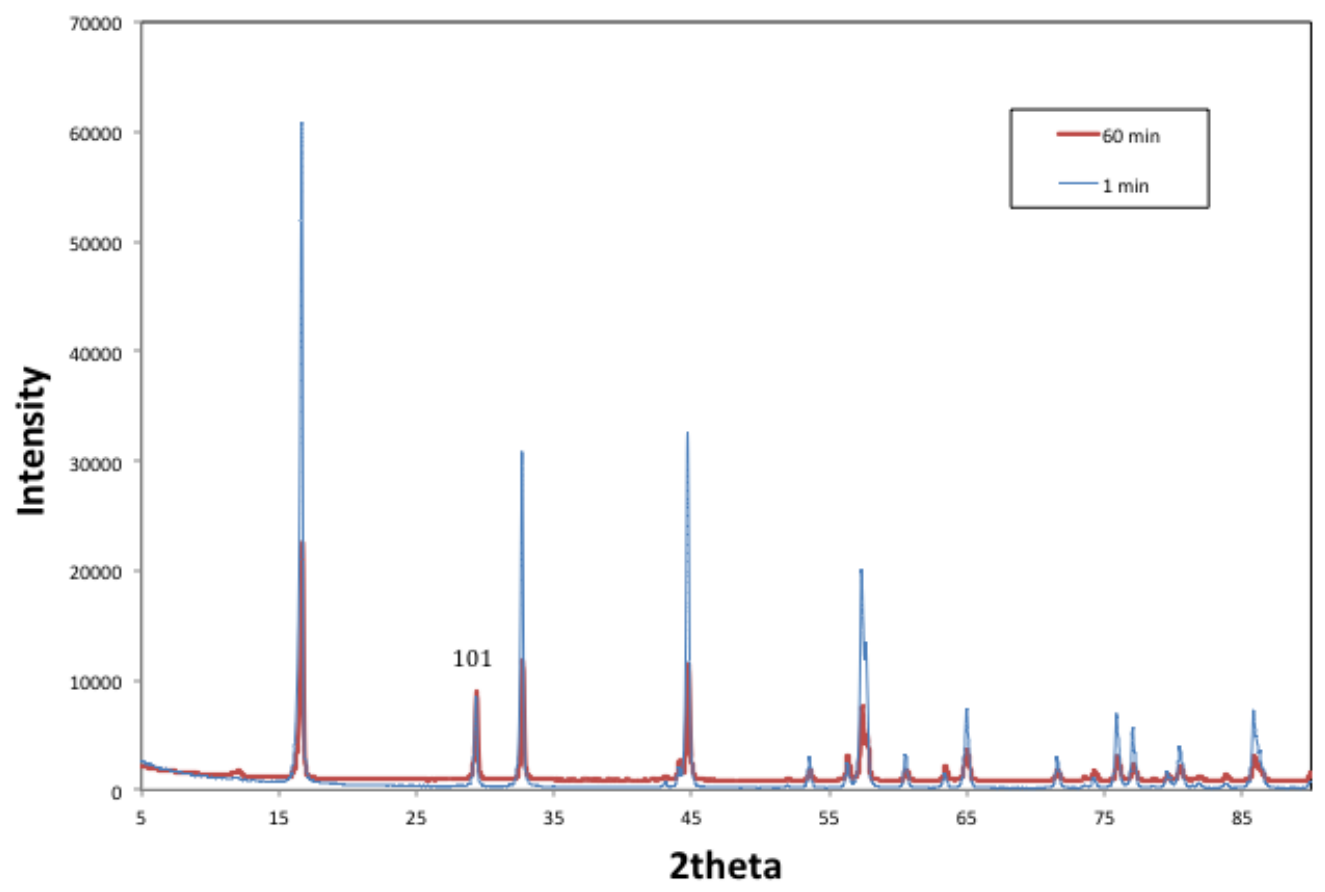

(b)

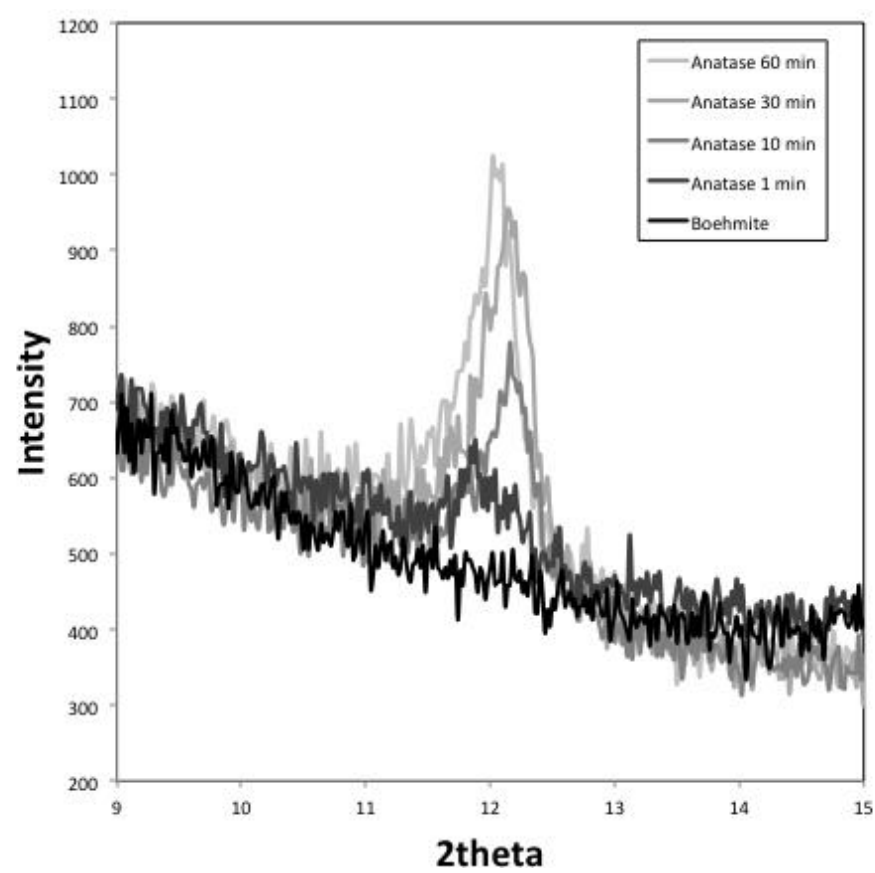

(c)

Figure 4. (a) XRD pattern of starting solids before digestion (b) XRD pattern of 'residues' - solids remaining after boehmite digestion in the presence of anatase at 1 and 60 minutes, the XRD patterns have been normalized to give the same anatase peak heights. Boehmite dissolves with time but does not completely disappear (c) sodium titanate 001 reflection versus time for solids remaining after boehmite digestion. The sodium titanate peak is increasing with time. 


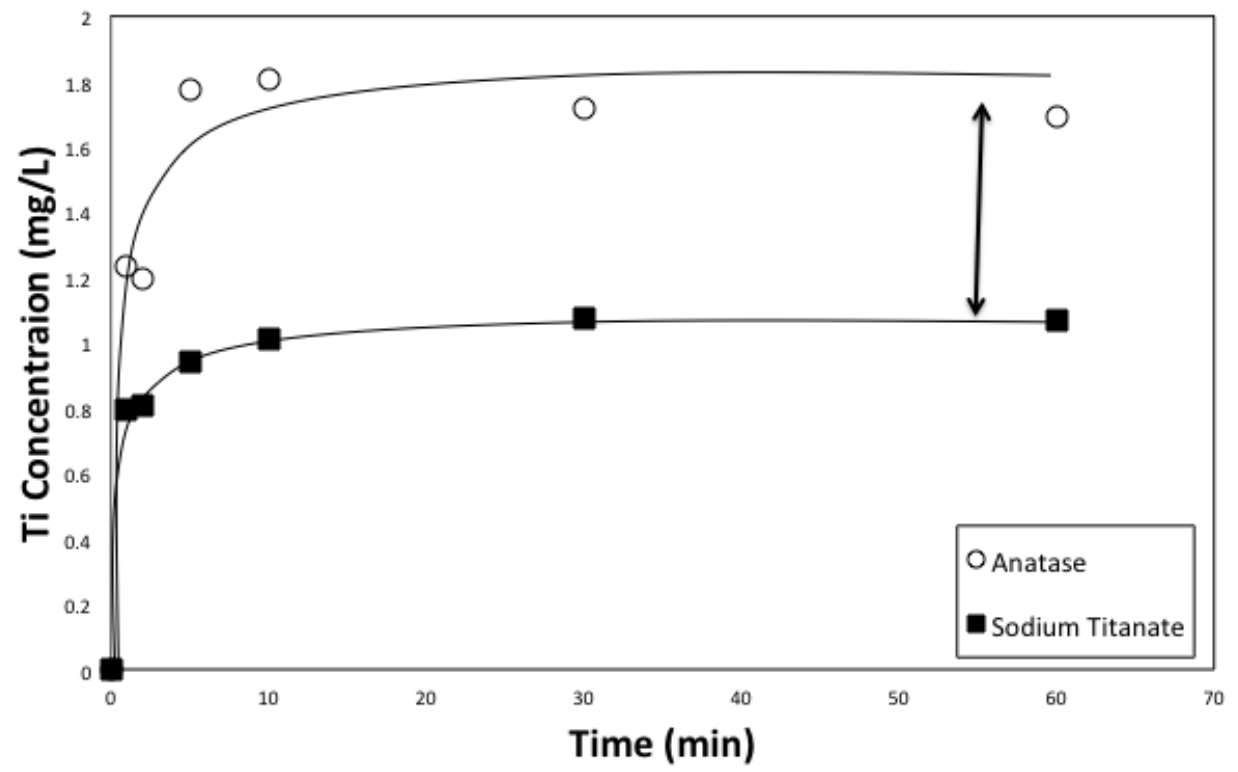

Figure 5. Concentration of $\mathrm{Ti}(\mathrm{mg} / \mathrm{L})$ in solution as measured by ICP for boehmite digestion in the presence of anatase or sodium titanate. Solubility of sodium titanate is lower than that of anatase. 


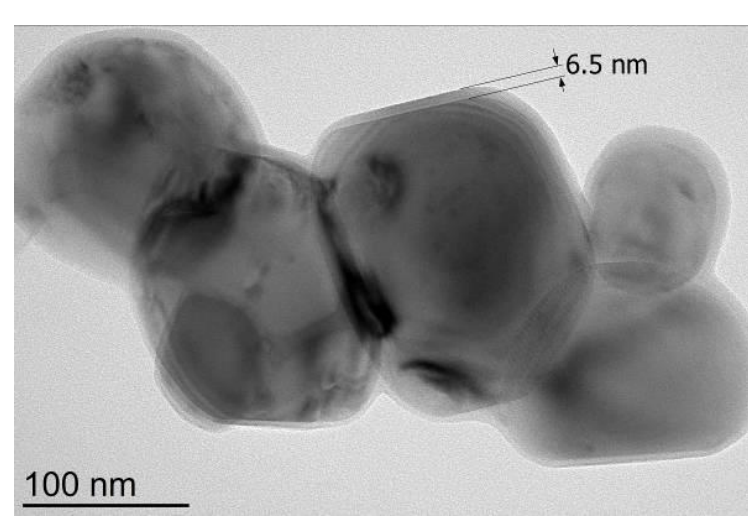

(a)

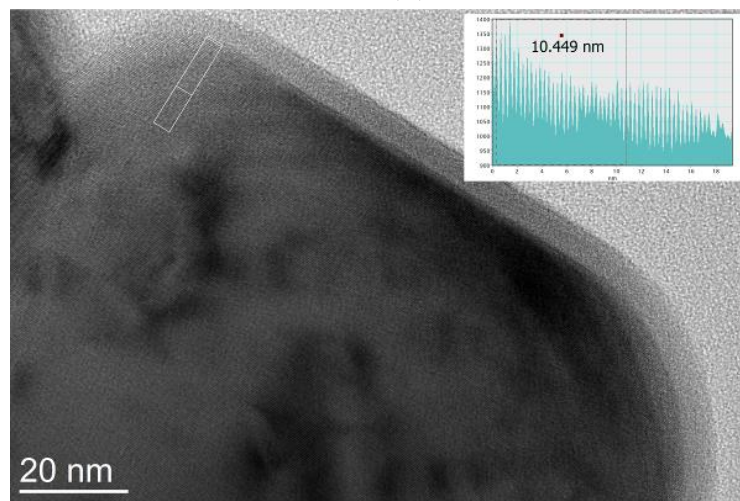

(c)

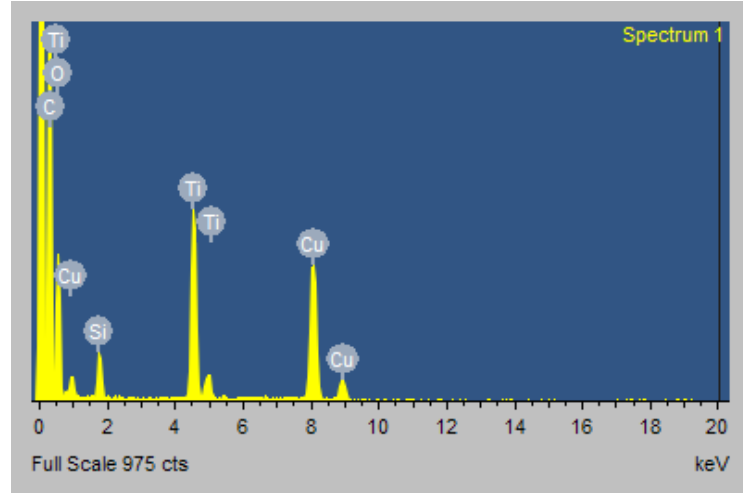

(e)

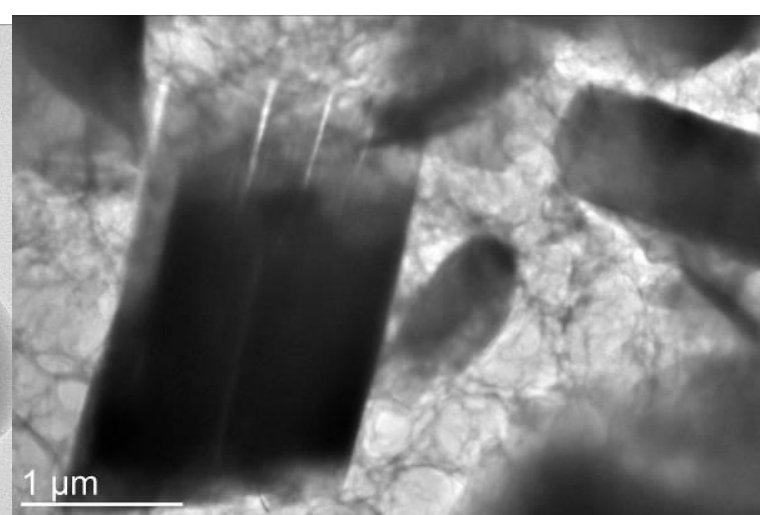

(b)

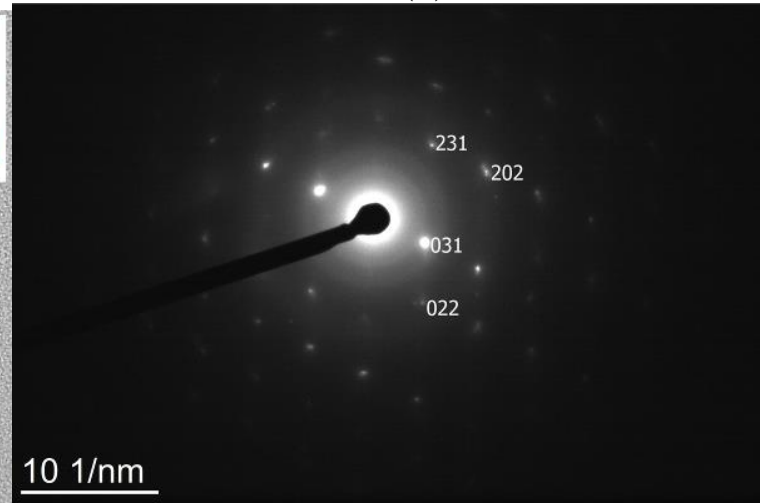

(d)

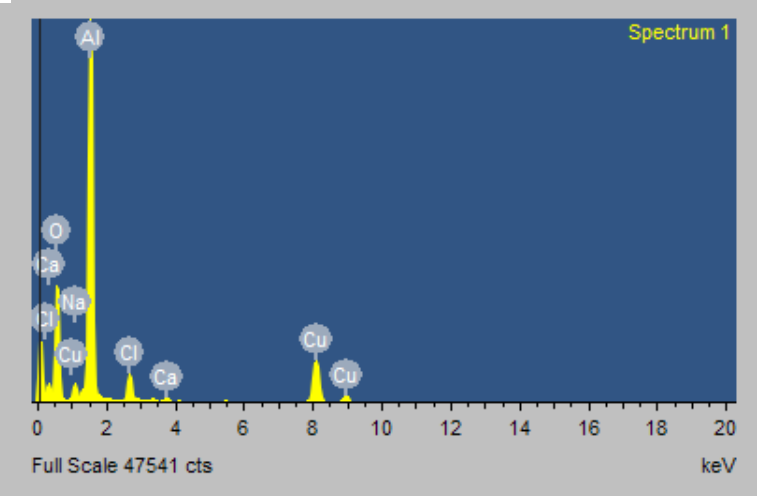

(f)

Figure 6. TEM characterisation of the staring materials. (a) TEM image of the anatase crystals; (b) TEM image of some typical boehmite crystals; (c) High resolution TEM images of anatase, 30 planes measured $104.49 \AA$ in the inset, matching with the d-spacing (3.50 ̊) of the anatase 101 plane; (d) SAED from the edge of the boehmite crystals; (e) EDS of the anatase crystals (no aluminium impurity); (f) EDS of the with boehmite crystals (no titanium impurity). Copper signal comes from TEM copper grid. 


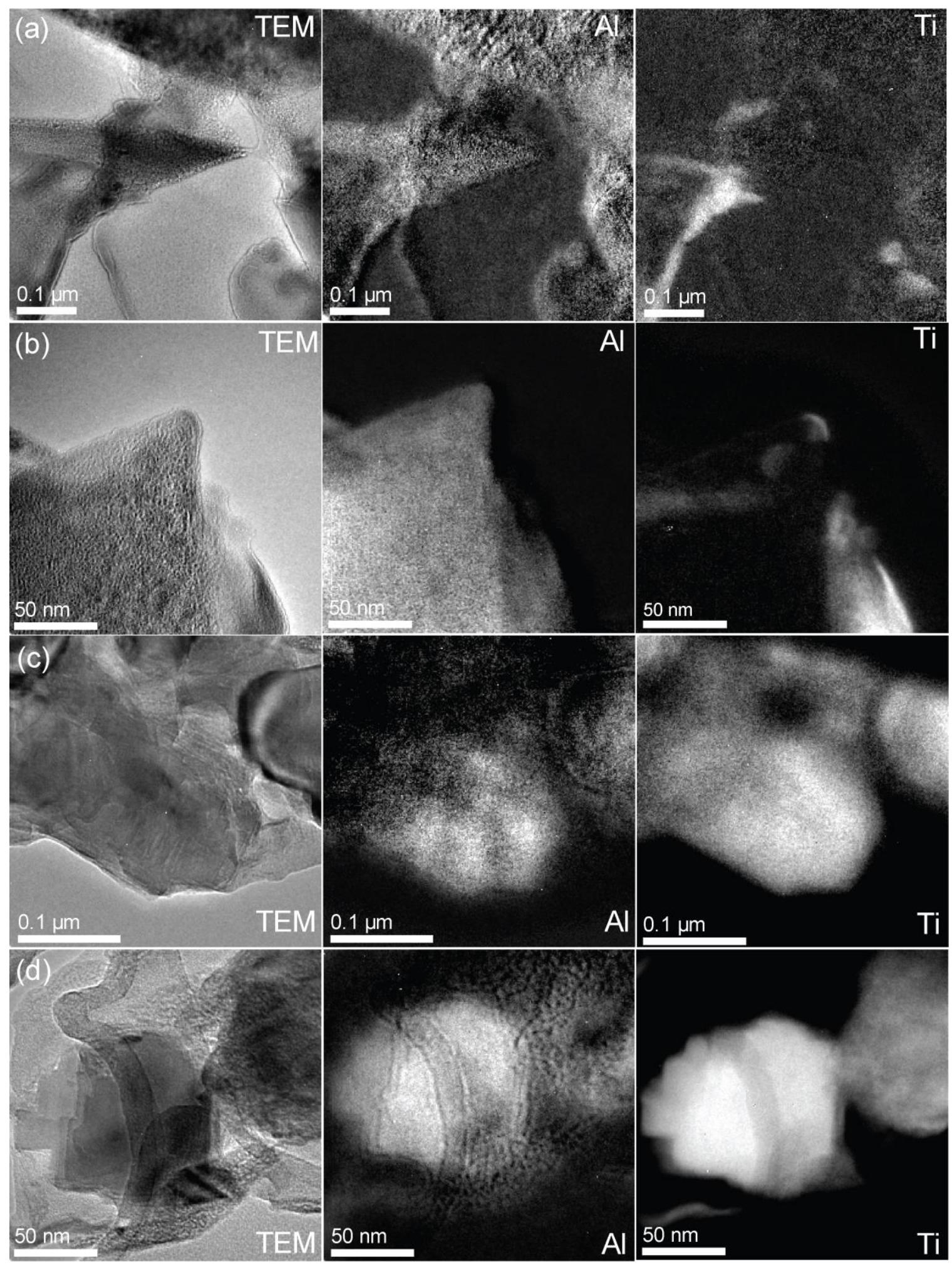

Figure 7. EFTEM images showing the aluminium and titanium distributions of the residual boehmite particles (a, b) and the residual anatase particles (c, d) after digestion in caustic for 60 minutes. Ti can be found on the surface of boehmite particles while Al can be found associated with some anatase particles. 


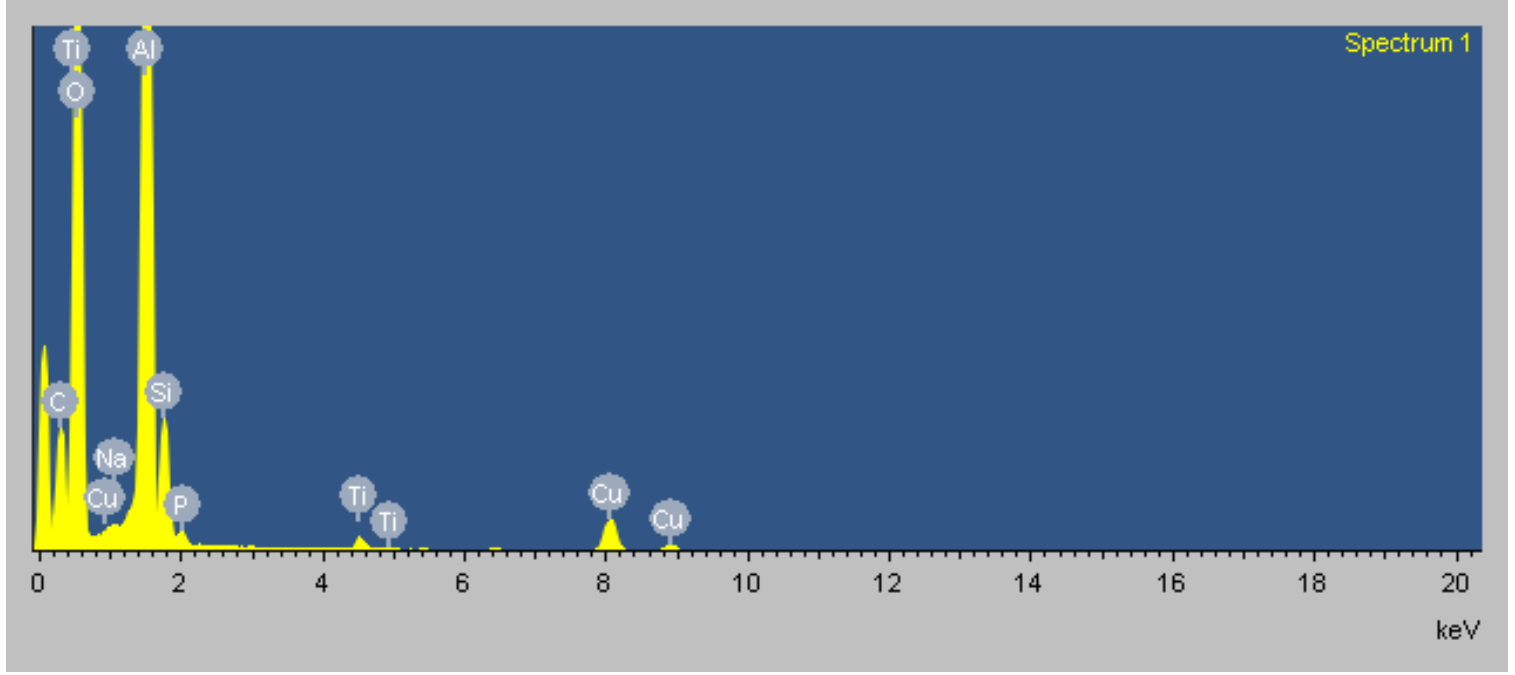

Figure 8. EDS of the boehmite particle shown in Figure 7(a).

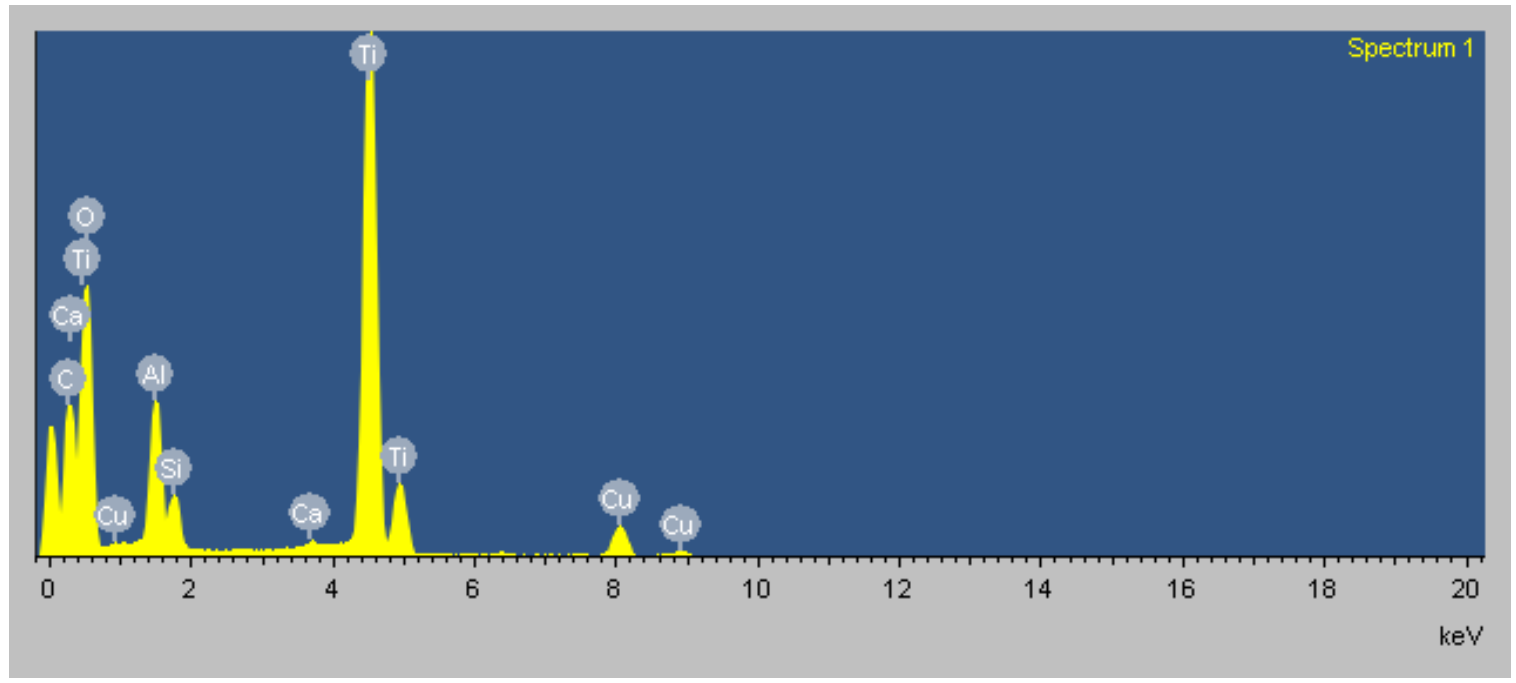

Figure 9. $\quad$ EDS of the anatase particle shown in Figure 7(c). 